\title{
Effects of the Antiepileptic Drugs Phenytoin, Gabapentin, and Levetiracetam on Bone Strength, Bone Mass, and Bone Turnover in Rats
}

\author{
Junkichi Kanda ${ }^{a}$ Nobuo Izumo, ${ }^{b}$ Yoshiko Kobayashi ${ }^{c}$ Kenji Onodera, ${ }^{d}$ Taketoshi Shimakura, ${ }^{e}$ \\ Noriaki Yamamoto, ${ }^{e, f}$ Hideaki E. Takahashi, ${ }^{e}$ and Hiroyuki Wakabayashi, ${ }^{* a}$ \\ ${ }^{a}$ Department of Clinical Pharmacotherapy, Faculty of Pharmaceutical Sciences, Niigata University of Pharmacy and \\ Applied Life Sciences; 265-1 Higashijima, Akiha-ku, Niigata 956-8603, Japan: ${ }^{b}$ General Health Medical Center, \\ Yokohama University of Pharmacy; 601 Matano-cho, Totsuka-ku, Yokohama 245-0066, Japan: ${ }^{c}$ Department of \\ Radiation Science, Yokohama University of Pharmacy; 601 Matano-cho, Totsuka-ku, Yokohama 245-0066, Japan: \\ ${ }^{d}$ Department of Clinical Pharmacotherapy and Pharmacy, Bethel Epilepsy Centre; 27-4 Minami, Hatamukaiyama, \\ Kitahase, Iwanuma, Miyagi 989-2455, Japan: ${ }^{e}$ Niigata Bone Science Institute; 761 Kizaki, Kita-ku, Niigata 950-3304, \\ Japan: and ${ }^{f}$ Division of Orthopedic Surgery, Niigata Rehabilitation Hospital; 761 Kizaki, Kita-ku, Niigata 950-3304, \\ Japan.
}

Received June 14, 2017; accepted August 25, 2017

Long-term treatment with antiepileptic drugs (AEDs) is accompanied by reduced bone mass that is associated with an increased risk of bone fractures. Although phenytoin has been reported to adversely influence bone metabolism, little is known pertaining to more recent AEDs. The aim of this study was to evaluate the effects of gabapentin or levetiracetam on bone strength, bone mass, and bone turnover in rats. Male Sprague-Dawley rats were orally administered phenytoin $(20 \mathrm{mg} / \mathrm{kg})$, gabapentin $(30$ or $150 \mathrm{mg} / \mathrm{kg})$, or levetiracetam $(50$ or $200 \mathrm{mg} / \mathrm{kg})$ daily for 12 weeks. Bone histomorphometric analysis of the tibia was performed and femoral bone strength was evaluated using a three-point bending method. Bone mineral density (BMD) of the femur and tibia was measured using quantitative computed tomography. Administration of phenytoin significantly decreased bone strength and BMD, which was associated with enhanced bone resorption. In contrast, treatment with gabapentin $(150 \mathrm{mg} / \mathrm{kg})$ significantly decreased bone volume and increased trabecular separation, as shown by bone histomorphometric analysis. Moreover, the bone formation parameters, osteoid volume and mineralizing surface, decreased after gabapentin treatment, whereas the bone resorption parameters, osteoclast surface and number, increased. Levetiracetam treatment did not affect bone strength, bone mass, and bone turnover. Our data suggested that gabapentin induced the rarefaction of cancellous bone, which was associated with decreased bone formation and enhanced bone resorption, and may affect bone strength and BMD after chronic exposure. To prevent the risk of bone fractures, patients prescribed a longterm administration of gabapentin should be regularly monitored for changes in bone mass.

Key words gabapentin; levetiracetam; osteoblast; osteoclast; rat

Epilepsy is a chronic neurological disorder characterized by recurrent seizures. ${ }^{1)}$ Pharmacotherapy with antiepileptic drugs (AEDs) is the mainstay of treatment for epilepsy and is effective in the prevention of seizures. Patients with epilepsy usually require long-term treatment with AEDs; thus, the prevention of side effects is important in the continuation of medication.

The bone is a metabolically active organ that undergoes continuous remodeling from bone resorption by osteoclasts and bone formation by osteoblasts. ${ }^{2}$ Under healthy conditions, the balance between bone formation and bone resorption is always uniform; thus, bone strength and bone mass are maintained. Certain pathological states and drugs affect normal bone remodeling, which can induce skeletal disorders, such as osteopenia or osteoporosis. ${ }^{3)}$ A serious side effect associated with long-term administration of AEDs is the elevated risk of bone fracture owing to a decrease in bone mass. ${ }^{4-7)}$ Patients taking AEDs are approximately 2-6 times more at risk of bone fracture than healthy individuals are. ${ }^{8,9)}$ Previous studies have demonstrated that treatment with phenytoin decreased bone mineral density (BMD) owing to enhanced bone resorption. ${ }^{10,11)}$ Currently, newly developed AEDs, including gabapentin, topiramate, lamotrigine, and levetiracetam, have been approved for clinical use. ${ }^{12)}$ Although traditional AEDs, such as phenytoin, continue to be used in patients with epilepsy, the prevalence of the new AEDs has increased. ${ }^{13)}$ Several new AEDs have been permitted for not only combination therapy with traditional AEDs, but also for monotherapy in patients with epilepsy. Moreover, newer AEDs are increasingly used for non-seizure indications, including peripheral neuropathy, bipolar disorder, and migraine headaches. Although it is expected that clinical use of newer AEDs will increase in future, there are few reports describing the effects of these AEDs on bone metabolism. The retrospective cohort study of Lee et al. ${ }^{14)}$ indicated a reduction in BMD in patients administered phenytoin, phenobarbital, and carbamazepine, but not in those administered gabapentin and levetiracetam. In contrast with those findings, a large retrospective cohort study revealed an elevated risk of bone fracture with the use of gabapentin for the treatment of epileptic patients. ${ }^{15)}$ The prospective study showed a significant bone loss in the femoral neck of elderly male patients administered gabapentin. ${ }^{16)}$ However, the mechanism of the effect of gabapentin on bone metabolism is uncertain and limited data are available with regard to the effects of the new AEDs on bone metabolism. Thus, in the present study, we investigated the effects of phenytoin, gabapentin, and levetiracetam on bone strength, bone mass, and bone turnover in rats. 


\section{MATERIALS AND METHODS}

Animals Male Sprague-Dawley rats (weight: 150-160g) were purchased from CLEA Japan Inc. (Tokyo, Japan). All animals were maintained at $22 \pm 2{ }^{\circ} \mathrm{C}, 55 \pm 5 \%$ humidity, under a 12-h light-dark cycle with ad libitum access to water and standard chow (MF; Oriental Yeast Co., Tokyo, Japan). All procedures were approved by the Animal Research Committee of Niigata University of Pharmacy and Applied Life Sciences (NUPALS) in accordance with the Japanese Government Animal Protection and Management Law, Japanese Government Notification on Feeding and Safekeeping of Animals, and the Guidelines for Animal Experiments in NUPALS.

Drugs Commercially available tablets of phenytoin (Sumitomo Dainippon Pharma, Tokyo, Japan), gabapentin (Pfizer Japan Inc., Tokyo, Japan), and levetiracetam (UCB Japan Co., Ltd., Tokyo, Japan) were obtained, uniformly pulverized, and suspended in $0.2 \%$ carboxymethylcellulose sodium (CMC-Na; Sigma-Aldrich, St. Louis, MO, U.S.A.) solution.

Experimental Procedure The animals were divided into six groups of nine animals each: control (vehicle; $0.2 \%$ CMC-Na solution), phenytoin $20 \mathrm{mg} / \mathrm{kg}$, gabapentin $30 \mathrm{mg} / \mathrm{kg}$, gabapentin $150 \mathrm{mg} / \mathrm{kg}$, levetiracetam $50 \mathrm{mg} / \mathrm{kg}$, and levetiracetam $200 \mathrm{mg} / \mathrm{kg}$. Drug doses were selected based on previous reports relevant to phenytoin, ${ }^{17,18)}$ gabapentin, ${ }^{19,20)}$ and levetiracetam. ${ }^{21)}$ Each drug was administered orally at a volume of $0.1 \mathrm{~mL} / 100 \mathrm{~g}$ body weight once per day for 12 weeks. Twentyfour hours after the final drug administration, the rats were anesthetized with $\mathrm{CO}_{2}$ and whole blood was collected. Serum was obtained by centrifugation of the blood sample at $1500 \times \boldsymbol{g}$ for $10 \mathrm{~min}$ and stored at $-80^{\circ} \mathrm{C}$ until measurement. The femur and tibia were dissected and soft tissues were removed.

Bone Strength Analysis The bone strength of the femoral mid-diaphysis was evaluated by a three-point bending method using a mechanical testing machine (EZ-S; Shimadzu, Tokyo, Japan). The femur was positioned on two supports placed $15 \mathrm{~mm}$ apart. The bending load was vertically applied to the mid-diaphysis with a crosshead speed of $1.0 \mathrm{~mm} / \mathrm{min}$ until bone fracture occurred. The load deformation curves were calculated using Operation software (Trapezium X; Shimadzu, Tokyo, Japan), and the maximum load, breaking energy, and stiffness were calculated directly from the load deformation curve.

Measurement of BMD The whole femur and tibia were measured using quantitative computed tomography (LaTheta LCT-100; Aloka, Tokyo, Japan) with a pixel size of $250 \times 250 \mu \mathrm{m}$ and a slice thickness of $1 \mathrm{~mm}$. Total BMD, cortical BMD, and trabecular BMD were calculated by using LaTheta software (ver. 1.31; Aloka, Tokyo, Japan).

Measurement of Serum Biochemical Markers Serum calcium levels were measured by using a commercial reagent (Denka Seiken, Tokyo, Japan) and an automatic analyzer (Hitachi7180; Hitachi, Tokyo, Japan). Serum 25-hydroxy vitamin D $(25(\mathrm{OH}) \mathrm{D})$ levels were measured by using the $25(\mathrm{OH})$ vitamin D direct day enzyme-linked immunosorbent assay (ELISA) kit (Immundiagnostik GmbH, Bensheim, Germany). Serum parathyroid hormone (PTH) levels were measured by using the Rat Intact PTH ELISA kit (Immutopics, San Clement, CA, U.S.A.). The levels of serum osteocalcin, a bone formation marker, were measured by using the Osteocalcin ELISA system for rats (General Electric Healthcare
Japan, Tokyo, Japan). The levels of serum tartrate-resistant acid phosphatase-5b (TRAP), a bone resorption marker, were measured using the Rat TRAP assay (SBA-Sciences, Oulu, Finland).

Bone Histomorphometric Analyses We prepared undecalcified specimens from the proximal tibia metaphysis. Briefly, the tibia was fixed with $70 \%$ ethanol for $7 \mathrm{~d}$, stained with Villanueva Bone Stain (basic fuchsin, fast green, orange G, and azure II; Merck, Darmstadt, Germany) in 70\% methanol for $7 \mathrm{~d}$, and embedded in methyl methacrylate resin. The resin blocks were sliced to a thickness of $5-\mu \mathrm{m}$ by using a microtome (Leica RM2255; Leica Inc., Nussloch, Germany). The measurements of all bone histomorphometric parameters were conducted in the secondary spongiosa region. To exclude the primary spongiosa, the measurement region was $0.7-2.7 \mathrm{~mm}$ distal to the lowest point of the growth plate and $0.2 \mathrm{~mm}$ from the lateral cortex. The bone histomorphometric measurements were performed by using a semiautomatic image analyzing system (Histometry RT CAMERA; System Supply, Nagano, Japan) at $250 \times$ magnification. The following bone structural parameters were obtained: bone volume per tissue volume (BV/TV), trabecular thickness (Tb.Th), trabecular number (Tb.N), and trabecular separation (Tb.Sp). The following bone formation parameters were obtained: osteoid surface per bone surface (OS/BS), osteoid volume per bone volume $(\mathrm{OV} / \mathrm{BV})$, and osteoblast surface per bone surface (Ob.S/BS). The following bone resorption parameters were obtained: eroded surface per bone surface (ES/BS), osteoclast number per bone surface (N.Oc/BS), and osteoclast surface per bone surface (Oc.S/BS). The dynamic parameters were measured using a double fluorescent labeling technique. For labeling, all rats were injected subcutaneously with $25 \mathrm{mg} / \mathrm{kg}$ tetracycline (Sigma-Aldrich) and $10 \mathrm{mg} / \mathrm{kg}$ calcein (Wako Pure Chemical Industries, Ltd., Osaka, Japan) at 5 and $2 \mathrm{~d}$ before sacrifice, respectively. The labeled surface, which reflected the calcification front at the time of tetracycline and calcein administration, was clearly visualized by using a fluorescent microscope (Olympus BX50; Olympus America Inc., Center Valley, PA, U.S.A.). The parameters of single- and doublelabeled surface (sLS and dLS, respectively) and inter-labels thickness and times (Ir.L.Th and Ir.L.t, respectively) were used in the calculation of the mineralizing surface per bone surface $[\mathrm{MS}(\mathrm{dLS}+\mathrm{sLS} / 2) / \mathrm{BS})]$ and the mineral apposition rate (MAR; Ir.L.Th/Ir.L.t). The standard bone histomorphometric nomenclature, symbols, and units were based on those described in the report of the American Society for Bone and Mineral Research (ASBMR) Histomorphometry Nomenclature Committee. ${ }^{22)}$

Statistical Analysis The data are presented as the mean \pm standard error of the mean (S.E.M.). The differences in the mean values between each of the groups were analyzed with one-way ANOVA followed by Dunnett's method of multiple comparisons. A value of $p<0.05$ was considered statistically significant.

\section{RESULTS}

Effects of Phenytoin, Gabapentin, and Levetiracetam on Bone Strength The following parameters of the femoral mid-diaphysis significantly decreased in the phenytoin-treated group compared with that in the control group: maximum load 
Table 1. Bone Strength of Femoral Mid-diaphysis

\begin{tabular}{|c|c|c|c|c|c|c|}
\hline & \multirow{2}{*}{ Control } & \multirow{2}{*}{$\begin{array}{l}\text { Phenytoin } \\
20 \mathrm{mg} / \mathrm{kg}\end{array}$} & \multicolumn{2}{|c|}{ Gabapentin } & \multicolumn{2}{|c|}{ Levetiracetam } \\
\hline & & & $30 \mathrm{mg} / \mathrm{kg}$ & $150 \mathrm{mg} / \mathrm{kg}$ & $50 \mathrm{mg} / \mathrm{kg}$ & $200 \mathrm{mg} / \mathrm{kg}$ \\
\hline Maximum load $(\mathrm{N})$ & $175 \pm 7.93$ & $147 \pm 9.99 *$ & $170 \pm 5.09$ & $168 \pm 6.06$ & $167 \pm 6.41$ & $165 \pm 3.17$ \\
\hline Breaking energy (N.mm) & $200 \pm 8.52$ & $160 \pm 8.45^{*}$ & $185 \pm 10.4$ & $181 \pm 9.86$ & $185 \pm 10.4$ & $180 \pm 10.1$ \\
\hline Stiffness (N/mm) & $222 \pm 8.01$ & $187 \pm 7.57 *$ & $216 \pm 4.14$ & $208 \pm 5.28$ & $213 \pm 5.34$ & $214 \pm 4.83$ \\
\hline
\end{tabular}

Data represents the mean \pm S.E.M. of nine rats. $* p<0.05$ vs. Control.

Table 2. Cortical, Trabecular, and Total Bone Mineral Density (BMD) of the Whole Femur and Tibia

\begin{tabular}{|c|c|c|c|c|c|c|}
\hline & \multirow{2}{*}{ Control } & \multirow{2}{*}{$\begin{array}{l}\text { Phenytoin } \\
20 \mathrm{mg} / \mathrm{kg}\end{array}$} & \multicolumn{2}{|c|}{ Gabapentin } & \multicolumn{2}{|c|}{ Levetiracetam } \\
\hline & & & $30 \mathrm{mg} / \mathrm{kg}$ & $150 \mathrm{mg} / \mathrm{kg}$ & $50 \mathrm{mg} / \mathrm{kg}$ & $200 \mathrm{mg} / \mathrm{kg}$ \\
\hline \multicolumn{7}{|l|}{ Whole femur } \\
\hline Cortical BMD $\left(\mathrm{mg} / \mathrm{cm}^{3}\right)$ & $1166 \pm 2.96$ & $1139 \pm 5.16^{*}$ & $1163 \pm 3.70$ & $1159 \pm 5.19$ & $1166 \pm 2.15$ & $1164 \pm 4.88$ \\
\hline Trabecular BMD $\left(\mathrm{mg} / \mathrm{cm}^{3}\right)$ & $600 \pm 3.89$ & $569 \pm 3.18 *$ & $593 \pm 5.71$ & $586 \pm 4.53$ & $599 \pm 8.70$ & $602 \pm 11.2$ \\
\hline Total BMD $\left(\mathrm{mg} / \mathrm{cm}^{3}\right)$ & $893 \pm 6.76$ & $846 \pm 7.07 *$ & $885 \pm 16.9$ & $874 \pm 7.85$ & $894 \pm 10.7$ & $892 \pm 7.50$ \\
\hline \multicolumn{7}{|l|}{ Whole tibia } \\
\hline Cortical BMD (mg/cm³ $)$ & $1190 \pm 1.81$ & $1167 \pm 3.81 *$ & $1189 \pm 3.89$ & $1182 \pm 6.31$ & $1189 \pm 2.66$ & $1190 \pm 2.78$ \\
\hline Trabecular BMD $\left(\mathrm{mg} / \mathrm{cm}^{3}\right)$ & $499 \pm 4.93$ & $472 \pm 7.66^{*}$ & $496 \pm 6.99$ & $488 \pm 4.55$ & $497 \pm 4.26$ & $495 \pm 6.19$ \\
\hline Total BMD $\left(\mathrm{mg} / \mathrm{cm}^{3}\right)$ & $916 \pm 7.32$ & $877 \pm 8.55^{*}$ & $913 \pm 8.18$ & $904 \pm 6.57$ & $915 \pm 12.0$ & $916 \pm 11.4$ \\
\hline
\end{tabular}

Data represents the mean \pm S.E.M. of nine rats. $* p<0.05 v$ s. Control.

Table 3. Serum Biochemical Markers

\begin{tabular}{|c|c|c|c|c|c|c|}
\hline & \multirow{2}{*}{ Control } & \multirow{2}{*}{$\begin{array}{l}\text { Phenytoin } \\
20 \mathrm{mg} / \mathrm{kg}\end{array}$} & \multicolumn{2}{|c|}{ Gabapentin } & \multicolumn{2}{|c|}{ Levetiracetam } \\
\hline & & & $30 \mathrm{mg} / \mathrm{kg}$ & $150 \mathrm{mg} / \mathrm{kg}$ & $50 \mathrm{mg} / \mathrm{kg}$ & $200 \mathrm{mg} / \mathrm{kg}$ \\
\hline Calcium (mg/dL) & $10.3 \pm 0.10$ & $10.1 \pm 0.08 *$ & $10.0 \pm 0.15$ & $10.1 \pm 0.18^{*}$ & $10.3 \pm 0.10$ & $10.2 \pm 0.07$ \\
\hline Osteocalcin (ng/dL) & $41.7 \pm 1.54$ & $38.9 \pm 2.26^{*}$ & $36.5 \pm 1.26$ & $32.7 \pm 1.82 *$ & $40.1 \pm 1.84$ & $39.2 \pm 1.19$ \\
\hline TRAP (U/L) & $13.3 \pm 1.68$ & $26.4 \pm 2.49^{*}$ & $17.0 \pm 1.32$ & $21.2 \pm 2.67 *$ & $13.7 \pm 0.64$ & $14.1 \pm 1.13$ \\
\hline $25(\mathrm{OH}) \mathrm{D}(\mathrm{mol} / \mathrm{L})$ & $215 \pm 10.2$ & $190 \pm 12.6^{*}$ & $209 \pm 11.5$ & $211 \pm 13.6^{*}$ & $215 \pm 17.8$ & $216 \pm 20.1$ \\
\hline PTH $(\mathrm{pg} / \mathrm{mL})$ & $41.5 \pm 7.00$ & $49.7 \pm 7.34 *$ & $41.2 \pm 8.22$ & $45.3 \pm 7.12 *$ & $41.7 \pm 5.44$ & $43.5 \pm 4.80$ \\
\hline
\end{tabular}

Data represents the mean \pm S.E.M. of nine rats. ${ }^{*} p<0.05$ s. Control. TRAP: tartrate-resistant acid phosphatase-5b, 25(OH)D: 25 -hydroxy vitamin D, PTH: parathyroid hormone.

(16\%), breaking energy (20\%), and stiffness (16\%) (Table 1). There were no significant differences in the bone strength parameters in the gabapentin- and levetiracetam-treated groups compared with the control group.

Effects of Phenytoin, Gabapentin, and Levetiracetam on BMD The phenytoin-treated group exhibited significantly decreased cortical BMD (2.3, 1.9\%), trabecular BMD (5.2, $5.3 \%)$, and total BMD $(5.2,4.3 \%)$ of the whole femur and tibia, respectively, compared with that in the control group (Table 2). However, no statistically significant differences in BMD were observed after treatment with gabapentin or levetiracetam compared with that in the control group.

Effects of Phenytoin, Gabapentin, and Levetiracetam on Serum Biochemical Markers The measurements of serum biochemical markers are summarized in Table 3. There were no significant differences in serum calcium levels, 25(OH)D levels, and PTH levels between all groups. However, serum TRAP levels significantly increased in the phenytoin-treated group compared with that in the control group. In the $150-\mathrm{mg} / \mathrm{kg}$ gabapentin-treated group, serum osteocalcin levels significantly decreased, whereas serum TRAP levels significantly increased compared with control values.

Bone Histomorphometric Evaluation The bone struc- tural parameters, according to bone histomorphometry of the proximal tibia metaphysis, are shown in Fig. 1. The phenytoin-treated and $150 \mathrm{mg} / \mathrm{kg}$ gabapentin-treated groups showed significantly reduced BV/TV (39, 18\%), Tb.Th (29, 13\%), and Tb.N (32, 14\%) and increased Tb.Sp (32, 20\%). The bone formation and bone resorption parameters based on bone histomorphometry are shown in Fig. 2. In the phenytoin-treated group, the bone resorption parameters ES/BS, Oc.S/BS, and N.Oc/BS significantly increased by approximately 69,85 , and $61 \%$, respectively. In the $150-\mathrm{mg} / \mathrm{kg}$ gabapentin-treated group, the bone formation parameters $\mathrm{OS} / \mathrm{BS}, \mathrm{OV} / \mathrm{BV}$, Ob.S/BS, MS/BS, and MAR significantly decreased by approximately $31,26,14,18$, and $13 \%$, respectively, whereas ES/BS, Oc.S/BS, and N.Oc/BS significantly increased by approximately 41,49 , and $40 \%$, respectively, compared with that in the control group. Typical micrographs of the slices (Fig. 3) were assessed by bone histomorphometric analysis of the control and $150-\mathrm{mg} / \mathrm{kg}$ gabapentin-treated groups. It was observed that bones in the $150 \mathrm{mg} / \mathrm{kg}$-gabapentin-treated group had a decreased osteoid surface, osteoid volume, and interlabel thickness compared with that in the control group. 
A

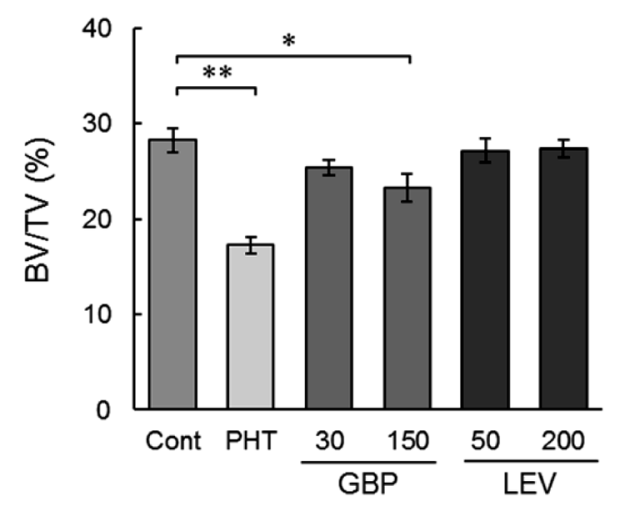

C

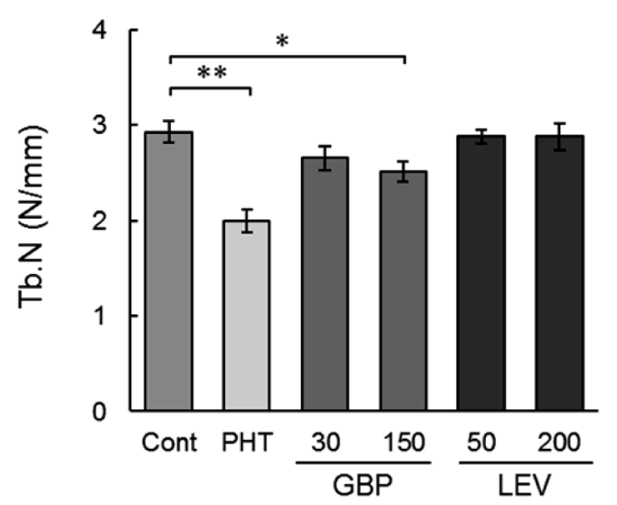

B

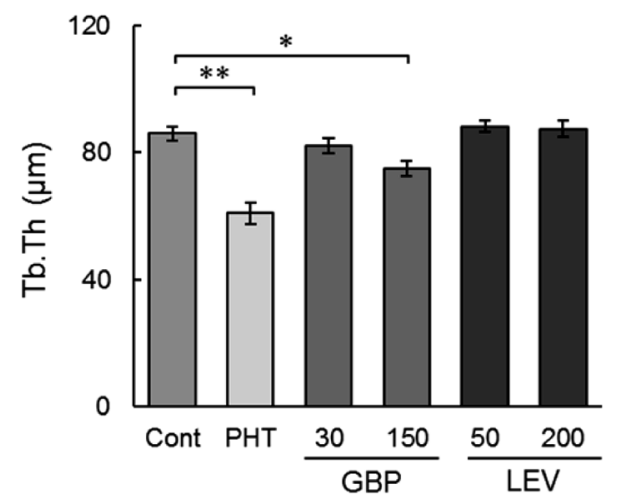

D

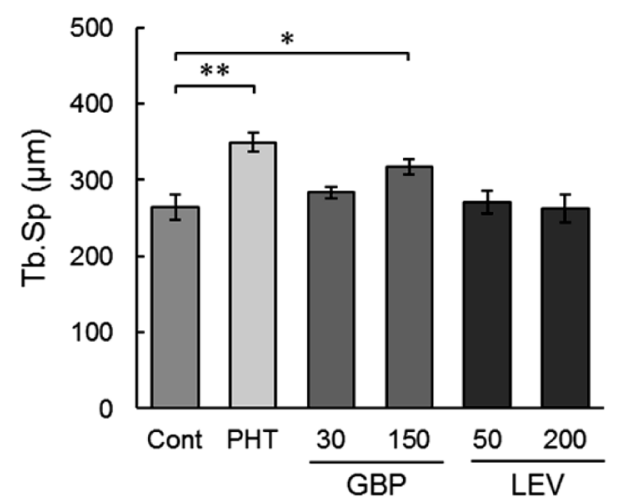

Fig. 1. Effects of Phenytoin, Gabapentin, and Levetiracetam on Bone Structural Parameters (A: BV/TV, B: Tb.Th, C: Tb.N, D: Tb.Sp) According to Bone Histomorphometry of the Proximal Tibia Metaphysis

Phenytoin (PHT; $20 \mathrm{mg} / \mathrm{kg}$ ), gabapentin (GBP; 30, $150 \mathrm{mg} / \mathrm{kg}$ ), and levetiracetam (LEV; 50, 200 mg/kg) were orally administered once per day for $12 \mathrm{weeks}$. The control group (Cont) was treated with vehicle $\left(0.2 \%\right.$ CMC-Na solution). Data represent the mean \pm S.E.M. of nine rats. ${ }^{*} p<0.05$, $* * p<0.01 v s$. Control. BV: bone volume, TV: tissue volume, Tb.Th: trabecular thickness, Tb.N: trabecular number, Tb.Sp: trabecular separation.

\section{DISCUSSION}

Long-term treatment with traditional AEDs has been shown to result in the decreased bone mass of patients with epilepsy and, consequently, an increased risk of bone fractures. ${ }^{4-7)}$ Indeed, it has been reported that long-term treatment with phenytoin resulted in decreased $\mathrm{BMD},{ }^{10,11}$ but few studies have examined the effects of the more recently developed newer AEDs on bone metabolism. In present study, we showed that the daily administration of phenytoin $20 \mathrm{mg} / \mathrm{kg}$ to rats for 12 weeks resulted in a significant decrease in bone strength and BMD, which was associated with enhanced bone resorption. Although treatment with gabapentin or levetiracetam in same experimental conditions resulted in no significant alteration of bone strength and $\mathrm{BMD}$, gabapentin at a dose of $150 \mathrm{mg} / \mathrm{kg}$ induced the rarefaction of cancellous bone in the proximal tibia metaphysis, which was associated with the suppression of bone formation and the enhancement of bone resorption. Previous studies assumed that one of the mechanism for bone loss by AEDs promoted the catabolism of $25(\mathrm{OH}) \mathrm{D}$ and $1,25(\mathrm{OH}) \mathrm{D}$. $^{23-25)}$ The resulting decrease in serum $25(\mathrm{OH}) \mathrm{D}$ and $1,25(\mathrm{OH}) \mathrm{D}$ levels leads to reduced calcium absorption, with consecutive secondary hyperparathyroidism, increased bone resorption, and accelerated bone loss. ${ }^{26)}$ In support of this hypothesis, several clinical studies have reported the elevation of serum PTH levels ${ }^{27)}$ and the reduction of serum $25(\mathrm{OH}) \mathrm{D}$ levels in patients treated with enzyme-inducing AEDs. ${ }^{28)}$ In contrast to this finding, several studies have observed no significant differences in these parameters. ${ }^{29,30)}$ In this study, we investigated the effect of AEDs on vitamin D metabolism, and found no significant difference in serum calcium, 25(OH)D, and PTH levels between the control group and the AED-treated groups. Although these results indicated that the AED-induced bone loss was not related to an abnormality in vitamin D metabolism, factors such as sex differences, species differences, and effects of duration of treatment need to be considered and investigated in the future.

Bone strength is defined by bone mass and bone quality ${ }^{31)}$; the maintenance of bone mass requires a certain balance between bone formation by osteoblasts and bone resorption by osteoclasts. ${ }^{2,32)}$ The term "bone quality" refers collectively to elements other than bone mass that affect bone fractures and is mainly defined by bone microstructure, bone turnover, microdamage, and bone mineralization. ${ }^{31)} \mathrm{A}$ meta-analysis concluded that the reduction in BMD was too small to explain the increased risk of bone fracture observed in patients with epilepsy who were prescribed traditional AEDs. ${ }^{33)}$ The assessment of bone quality is important in the evaluation of the effects of AEDs on bone metabolism. In this study, we performed an analysis of the cancellous bone microstructure and the abilities of the bone mineralization by the bone histomorphometry in order to evaluate the effect of AEDs on bone 
A

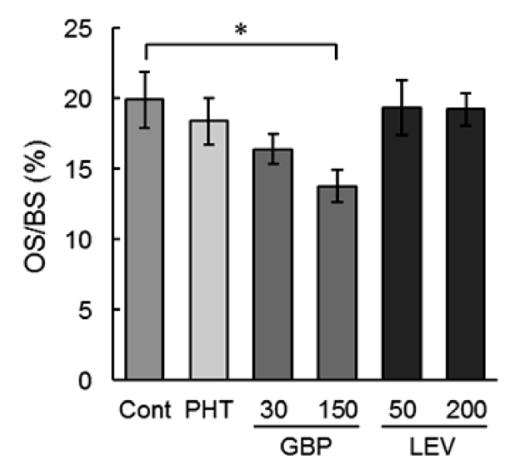

D

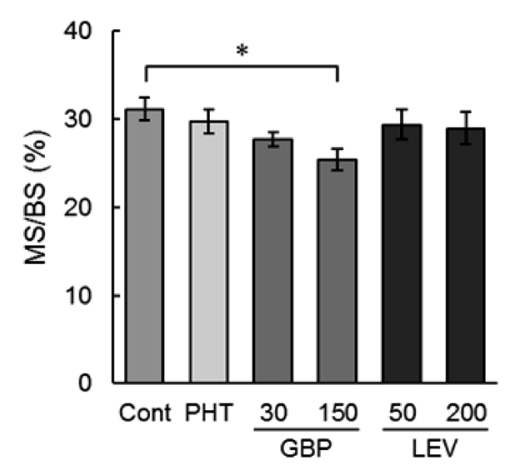

G

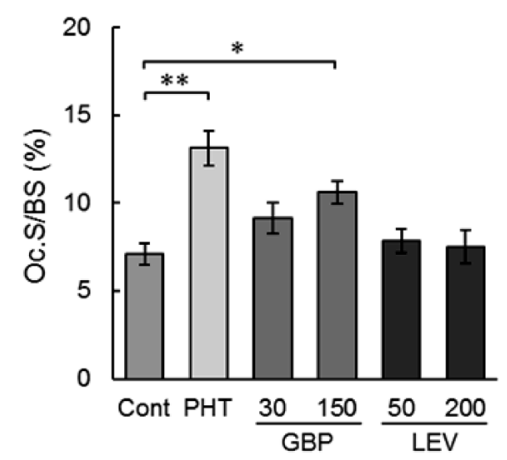

B

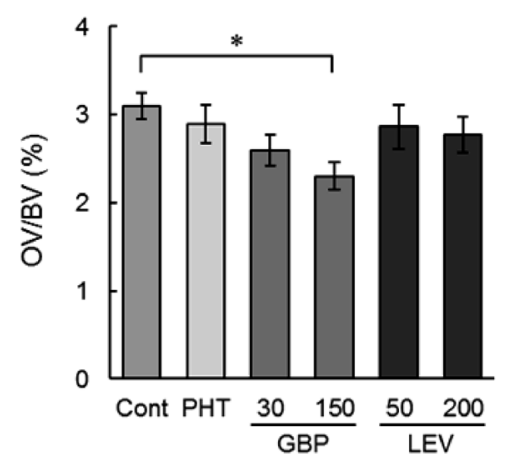

E
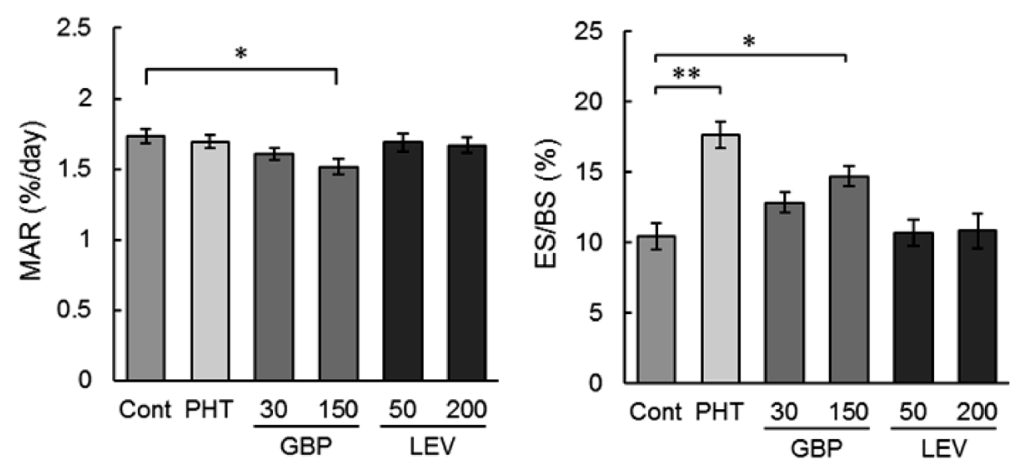

$\mathrm{H}$

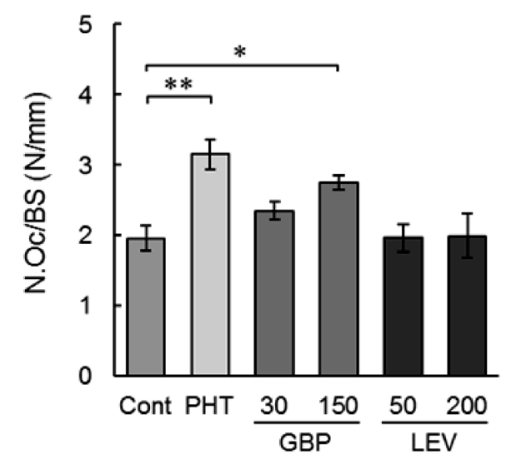

Fig. 2. Effects of Phenytoin, Gabapentin, and Levetiracetam on Bone Formation Parameters (A: OS/BS, B: OV/BV, C: Ob.S/BS, D: MS/BS, E: MAR) and Bone Resorption Parameters (F: ES/BS, G: Oc.S/BS, H: N.Oc/BS) According to Bone Histomorphometry of the Proximal Tibia Metaphysis

Phenytoin (PHT; $20 \mathrm{mg} / \mathrm{kg}$ ), gabapentin (GBP; 30, $150 \mathrm{mg} / \mathrm{kg}$ ), and levetiracetam (LEV; 50, 200 mg/kg) were orally administered once per day for $12 \mathrm{weeks}$. The control group (Cont) was treated with vehicle $\left(0.2 \%\right.$ CMC-Na solution). Data represent the mean \pm S.E.M. of nine rats. ${ }^{*} p<0.05, * * p<0.01 v s$. Control. OS: osteoid surface, BS: bone surface, OV: osteoid volume, BV: bone volume, Ob.S: osteoblast surface, MS: mineralizing surface, MAR: mineral apposition rate, ES: eroded surface, Oc.S: osteoclast surface, N.Oc: osteoclast number.

quality. As treatment with phenytoin and gabapentin at a dose at $150 \mathrm{mg} / \mathrm{kg}$ significantly decreased BV/TV, Tb.N, and Tb.Th associated with increased Tb.Sp, it was suggested that these drugs caused deterioration of the cancellous bone microstructure. Bone resorption parameters, such as ES/BS, Oc.S/BS, and N.Oc/BS, significantly increased in the phenytoin-treated and $150 \mathrm{mg} / \mathrm{kg}$ gabapentin-treated group. In contrast, bone formation parameters, such as OS/BS, OV/BV, Ob.S/BS, MS/BS, and MAR, significantly decreased in the $150 \mathrm{mg} / \mathrm{kg}$ gabapentin-treated group. OS/BS and $\mathrm{OV} / \mathrm{BV}$ are an indication of the ratio of uncalcified bone volume, whereas MS/BS and MAR are considered an index of osteoblast differentiation and proliferation. ${ }^{34)}$ Therefore, these results suggested that gabapentin may reduce the abilities of osteoblast and mineralization. In this study, the effects of AEDs on bone resorption and bone formation were also observed through the evaluation of serum biochemical markers. Collectively, we have demonstrated that phenytoin significantly decreased bone mass owing to the enhancement of bone resorption without influencing bone formation, which was in accordance with previous reports. ${ }^{10,11)}$ 

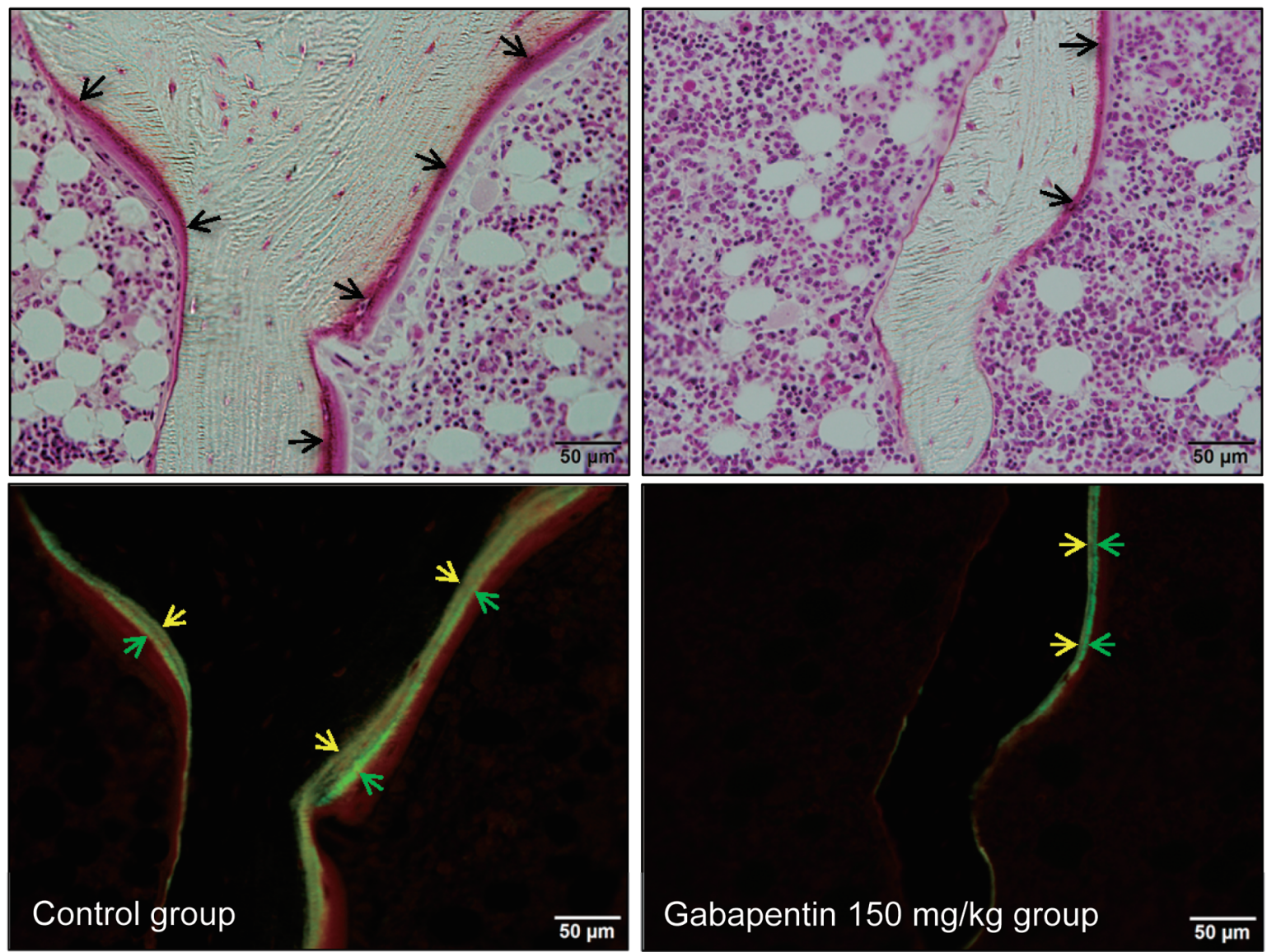

Fig. 3. Typical Micrographs of the Slices Assessed by Bone Histomorphometry under Natural Light (Upper Photos) and Fluorescence (Lower Photos)

In the upper photos, the osteoid surface is indicated by the black arrows. In the lower photos, the labeled surface with tetracycline and calcein are indicated by the yellow arrow and the green arrow, respectively (Villanueva Bone Stain, $\times 200$ ).

Furthermore, it was suggested that gabapentin significantly decreased bone volume owing to the uncoupling of bone turnover by the suppression of bone formation and the enhancement of bone resorption.

In our study, treatment with levetiracetam did not affect BMD or bone metabolism. This was in agreement with a recent clinical study on the effect of levetiracetam on bone metabolism, which indicated that it did not affect bone strength or bone metabolism in epileptic patients. ${ }^{35)}$ In contrast to phenytoin and gabapentin, levetiracetam does not affect bone metabolism. Therefore, the difference in mechanism of action of AEDs and the differences in their effects on bone metabolism should be clarified in the future.

The limitation of this study was the short treatment period that was used to evaluate the effects of gabapentin on bone metabolism in rats. There was no evidence that gabapentin affected bone strength or BMD in this study. However, the drug was associated with deterioration of the cancellous bone microstructure owing to decreased bone formation and increased bone resorption after 12 weeks, which may lead to a long-term effect on bone strength or BMD. Therefore, future studies are needed to assess the long-term effects of gabapentin.

In conclusion, we demonstrated that the administration of phenytoin $20 \mathrm{mg} / \mathrm{kg}$ to rats for 12 weeks significantly decreased bone strength and BMD, which was associated with enhanced bone resorption, whereas treatment with newer AEDs, such as gabapentin or levetiracetam, did not significantly affect bone strength and BMD. However, it was clear that the in vivo administration of gabapentin led to an imbalance in bone remodeling, in part by the suppression of bone formation and the enhancement of bone resorption, which led to the rarefaction of cancellous bone. In the current therapy of epilepsy, gabapentin is generally used in combination with another AED, such as phenytoin, and the drugs are prescribed for an extended period. Therefore, the effects of gabapentin on bone metabolism may additively increase the bone fragility induced by another AED. Hence, these findings suggest that patients receiving gabapentin over a prolonged period should be regularly monitored for changes in bone mass to prevent the risk of bone fractures.

Conflict of Interest The authors declare no conflict of interest.

\section{REFERENCES}

1) Kotsopoulos IA, van Merode $T$, Kessels FG, de Krom MC, Knottnerus JA. Systematic review and meta-analysis of incidence studies of epilepsy and unprovoked seizures. Epilepsia, 43, 14021409 (2002).

2) Raisz LG. Physiology and pathophysiology of bone remodeling. Clin. Chem., 45, 1353-1358 (1999).

3) Rodan GA, Martin TJ. Therapeutic approaches to bone diseases. Science, 289, 1508-1514 (2000).

4) Pack A. Bone health in people with epilepsy: is it impaired and what are the risk factors? Seizure, 17, 181-186 (2008).

5) Nissen-Meyer LS, Svalheim S, Tauboll E, Gjerstad L, Reinholt FP, 
Jemtland R. How can antiepileptic drugs affect bone mass, structure and metabolism? Lessons from animal studies. Seizure, 17, 187-191 (2008).

6) Swanton J, Simister R, Altmann D, Watts H, Keen R, Duncan JS, Koepp MJ. Bone mineral density in institutionalised patients with refractory epilepsy. Seizure, 16, 538-541 (2007).

7) Nicholas JM, Ridsdale L, Richardson MP, Grieve AP, Gulliford MC. Fracture risk with use of liver enzyme inducing antiepileptic drugs in people with active epilepsy: cohort study using the general practice research database. Seizure, 22, 37-42 (2013).

8) Mattson RH, Gidal BE. Fractures, epilepsy, and antiepileptic drugs. Epilepsy Behav., 5 (Suppl. 2), S36-S40 (2004).

9) Sheth RD, Gidal BE, Hermann BP. Pathological fractures in epilepsy. Epilepsy Behav., 9, 601-605 (2006).

10) Takahashi A, Onodera K, Shinoda H, Mayanagi H. Phenytoin and its metabolite, 5-(4-hydroxyphenyl)-5-phenylhydantoin, show bone resorption in cultured neonatal mouse calvaria. Jpn. J. Pharmacol., 82, 82-84 (2000)

11) Moro-Alvarez MJ, Diaz Curiel M, de la Piedra C, Marinoso ML, Carrascal MT. Bone disease induced by phenytoin therapy: clinical and experimental study. Eur. Neurol., 62, 219-230 (2009).

12) Reimers A. New antiepileptic drugs and women. Seizure, 23, 585591 (2014).

13) Berlowitz DR, Pugh MJ. Pharmacoepidemiology in communitydwelling elderly taking antiepileptic drugs. Int. Rev. Neurobiol., 81, 153-163 (2007)

14) Lee R, Lyles K, Sloane R, Colon-Emeric C. The association of newer anticonvulsant medications and bone mineral density. Endocr. Pract., 14 (aop), 1-22 (2012).

15) Jetté N, Lix LM, Metge CJ, Prior HJ, McChesney J, Leslie WD. Association of antiepileptic drugs with nontraumatic fractures: a population-based analysis. Arch. Neurol., 68, 107-112 (2011)

16) Ensrud KE, Walczak TS, Blackwell TL, Ensrud ER, Barrett-Connor E, Orwoll ES. Antiepileptic drug use and rates of hip bone loss in older men: a prospective study. Neurology, 71, 723-730 (2008).

17) Onodera K, Takahashi A, Mayanagi H, Wakabayashi H, Kamei J, Shinoda H. Phenytoin-induced bone loss and its prevention with alfacalcidol or calcitriol in growing rats. Calcif. Tissue Int., 69, 109-116 (2001).

18) Hoshino M, Ikarashi N, Hirobe R, Hayashi M, Hiraoka H, Yokobori K, Ochiai T, Kusunoki Y, Kon R, Tajima M, Ochiai W, Sugiyama $\mathrm{K}$. Effects of menthol on the pharmacokinetics of triazolam and phenytoin. Biol. Pharm. Bull., 38, 454-460 (2015).

19) Dominick MA, Robertson DG, Bleavins MR, Sigler RE, Bobrowski WF, Gough AW. Alpha 2u-globulin nephropathy without nephrocarcinogenesis in male Wistar rats administered 1-(aminomethyl)cyclohexaneacetic acid. Toxicol. Appl. Pharmacol., 111, 375-387 (1991).

20) Ling Q, Liu M, Wu MX, Xu Y, Yang J, Huang HH, Yu CX. Anti-allodynic and neuroprotective effects of koumine, a Benth alkaloid, in a rat model of diabetic neuropathy. Biol. Pharm. Bull., 37, 858-864 (2014).

21) Gower AJ, Noyer M, Verloes R, Gobert J, Wulfert E. ucb L059, a novel anti-convulsant drug: pharmacological profile in animals.
Eur. J. Pharmacol., 222, 193-203 (1992).

22) Dempster DW, Compston JE, Drezner MK, Glorieux FH, Kanis JA, Malluche H, Meunier PJ, Ott SM, Recker RR, Parfitt AM. Standardized nomenclature, symbols, and units for bone histomorphometry: a 2012 update of the report of the ASBMR Histomorphometry Nomenclature Committee. J. Bone Miner. Res., 28, 2-17 (2013).

23) Collins N, Maher J, Cole M, Baker M, Callaghan N. A prospective study to evaluate the dose of vitamin D required to correct low 25-hydroxyvitamin D levels, calcium, and alkaline phosphatase in patients at risk of developing antiepileptic drug-induced osteomalacia. Q. J. Med., 78, 113-122 (1991).

24) Pascussi JM, Robert A, Nguyen M, Walrant-Debray O, Garabedian M, Martin P, Pineau T, Saric J, Navarro F, Maurel P, Vilarem MJ. Possible involvement of pregnane X receptor-enhanced CYP24 expression in drug-induced osteomalacia. J. Clin. Invest., 115, 177-186 (2005).

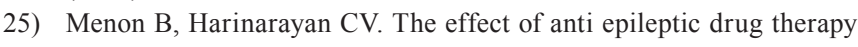
on serum 25-hydroxyvitamin D and parameters of calcium and bone metabolism-a longitudinal study. Seizure, 19, 153-158 (2010).

26) Fitzpatrick LA. Pathophysiology of bone loss in patients receiving anticonvulsant therapy. Epilepsy Behav., 5 (Suppl. 2), S3-S15 (2004).

27) Välimäki MJ, Tiihonen $M$, Laitinen $K$, Tähtelä R, Kärkkäinen $M$, Lamberg-Allardt C, Mäkelä P, Tunninen R. Bone mineral density measured by dual-energy X-ray absorptiometry and novel markers of bone formation and resorption in patients on antiepileptic drugs. J. Bone Miner. Res., 9, 631-637 (1994).

28) Pack AM, Morrell MJ, Randall A, McMahon DJ, Shane E. Bone health in young women with epilepsy after one year of antiepileptic drug monotherapy. Neurology, 70, 1586-1593 (2008).

29) Tsukahara H, Kimura K, Todoroki Y, Ohshima Y, Hiraoka M, Shigematsu Y, Tsukahara Y, Miura M, Mayumi M. Bone mineral status in ambulatory pediatric patients on long-term anti-epileptic drug therapy. Pediatr. Int., 44, 247-253 (2002).

30) Andress DL, Ozuna J, Tirschwell D, Grande L, Johnson M, Jacobson AF, Spain W. Antiepileptic drug-induced bone loss in young male patients who have seizures. Arch. Neurol., 59, 781-786 (2002).

31) NIH Consensus Development Panel on Osteoporosis Prevention, Diagnosis, and Therapy. Osteoporosis prevention, diagnosis, and therapy. JAMA, 285, 785-795 (2001).

32) Manolagas SC. Birth and death of bone cells: basic regulatory mechanisms and implications for the pathogenesis and treatment of osteoporosis. Endocr. Rev., 21, 115-137 (2000).

33) Vestergaard P. Epilepsy, osteoporosis and fracture risk - a metaanalysis. Acta Neurol. Scand., 112, 277-286 (2005).

34) Recker RR, Kimmel DB, Dempster D, Weinstein RS, Wronski TJ, Burr DB. Issues in modern bone histomorphometry. Bone, 49, 955-964 (2011).

35) Koo DL, Joo EY, Kim D, Hong SB. Effects of levetiracetam as a monotherapy on bone mineral density and biochemical markers of bone metabolism in patients with epilepsy. Epilepsy Res., 104, 134-139 (2013). 